\title{
SIMULATION OF FLUID'S AERATION: IMPLEMENTATION OF A NUMERICAL MODEL IN AN OPEN SOURCE ENVIRONMENT
}

\author{
MARCO NICOLA MASTRONE \& FRANCO CONCLI \\ Free University of Bolzano, Italy
}

\begin{abstract}
Multiphase flows often involve additional complex physical phenomena to be considered, for example cavitation and aeration. These can arise in several applications as gear pumps, propellers, and gearboxes. In order to properly design such hydraulic systems, these aspects should be carefully considered in the design phase. In fact, these phenomena can lead to lower efficiency, excessive noise, and a rapid degradation of the mechanical system. While for cavitation analytical and numerical models are already available, aeration, which refers to the entrainment of air in another fluid in form of bubbles and/or foam, is more difficult to be quantified. Even experimentally niche equipment is required. However, thanks to recent developments in computer science, it is possible to approach these complex multiphase flows exploiting simulations' tools. In this paper, a numerical approach based on Computational Fluid Dynamics (CFD) that includes the aeration phenomenon is presented. A new solver that accounts for the air entrapment in the fluid was implemented in the open source environment OpenFOAM ${ }^{\circledR}$. The solver was applied on a test case to verify its behaviour and compared to a standard multiphase solver. The qualitative analysis suggests that the solver is capable of considering the amount of air entrapped in the fluid, thus opening a new way in the study of aeration.
\end{abstract}

Keywords: aeration, CFD, multiphase modelling, OpenFOAM.

\section{INTRODUCTION}

Thanks to continuous technological developments in computer science, simulation software packages have gained their consideration not only among academic researchers, but also in industries. The advantages introduced by virtual prototyping, namely the reduction of time and costs with respect to real prototypes, have encouraged engineers to exploit these new opportunities. Complex multiphase problems can be solved numerically exploiting dedicated software where proper boundary conditions are assigned to each part of the computational domain. The study of multiphase problems is of great interest for real engineering applications as lubrication mechanisms, design of hydraulic systems, and in general every condition where more fluids are present simultaneously.

Standard multiphase solvers are not capable of considering additional physical phenomena, as cavitation and aeration, which play a determinant role in several operating conditions and must be carefully considered for a proper design of a system to avoid efficiency reduction, excessive noise, and the rapid degradation of the mechanical system. In fact, in order to include also possible phase changes (cavitation) and air entrainment in another fluid in form of bubbles and/or foam (aeration), the conservation equations of fluid dynamics must be modified by adding a source term that accounts for the new phenomena. While works dealing with standard multiphase solvers [1]-[13] and the simulation of cavitation in the design of mechanical components are already available [14]-[19], the aeration has been poorly touched from a numerical point of view. The development of a numerical solver that includes the air trapping in another fluid can lead to significant advancements in the correct modelling of real physical problems. Indeed, in hydraulic systems, the presence of air can cause the premature degradation of the lubricant and the increase of wear of mechanical components. Moreover, aeration has a severe impact on the 
heat transfer capabilities of the lubricant, thus reducing the global efficiency of the system and the heat dissipation capability, which, in turn, is responsible for overheating and possible premature failures.

In order to quantify the level of aeration in various operating conditions, niche and expensive equipment is required [20]-[23]. Hence, CFD offers the opportunity to investigate this complex phenomenon numerically. A numerical approach to calculate the air entrainment in a fluid was proposed by Cerne et al. [24], who established an interface tracking algorithm based on a two-fluid model formulation [25]. Yan and Che [26] extended the previous model for three fluid phases. A combination of Eulerian approach and Volume of Fluid (VOF) was tested by Wardle and Weller [27]. Ma et al. [28] reformulated the source term proposed by Sene [29] by including the turbulence intensity and tested their model on an hydraulic jump case [30], a plunging jet [31] and flow around a ship [32]. Several experiments related to plunging jet [33]-[36] have been exploited to study aeration, since, due to the energy dissipation, air bubbles are generated at the water free surface. In this work, a solver that considers aeration is implemented in the open source software OpenFOAM ${ }^{\circledR}$ and applied to two test cases. The promising results suggest that numerical tools can offer an opportunity to investigate conditions where experimental data might be difficult to obtain due to the complexity of the system and the required equipment.

\section{MATERIALS AND METHODS}

\subsection{Mathematical description}

CFD codes are based on the solution of three governing equations: mass, momentum, and energy conservation. In this study, the problem was modelled as isothermal. Therefore, the energy equation was not included in the calculation. In this way, the solution is limited to the mass and momentum equations which can be written as:

$$
\begin{gathered}
\frac{\partial \rho\left\langle\boldsymbol{u}_{i}\right\rangle}{\partial x_{i}}=0 \\
\frac{\partial\left(\rho\left\langle\boldsymbol{u}_{i}\right\rangle\right)}{\partial t}+\frac{\partial\left(\rho\left\langle\boldsymbol{u}_{i}\right\rangle\left\langle\boldsymbol{u}_{i}\right\rangle\right)}{\partial x_{j}}=-\frac{\partial\langle p\rangle}{\partial x_{i}}+\frac{\partial}{\partial x_{j}}\left[\mu\left(\frac{\partial\left\langle\boldsymbol{u}_{i}\right\rangle}{\partial x_{j}}+\frac{\partial\left\langle\boldsymbol{u}_{j}\right\rangle}{\partial x_{i}}\right)\right]-\frac{\partial \tau_{i j}}{\partial x_{j}}
\end{gathered}
$$

The term $\tau_{i j}$ is the Reynolds term and is expressed exploiting the eddy viscosity $\left(\mu_{t}\right)$ hypothesis:

$$
\begin{gathered}
-\tau_{i j}=\mu_{t}\left(\frac{\partial\left\langle\boldsymbol{u}_{i}\right\rangle}{\partial x_{j}}+\frac{\partial\left\langle\boldsymbol{u}_{j}\right\rangle}{\partial x_{i}}\right)-\frac{2}{3} \rho \delta_{i j} k . \\
\mu_{t}=C_{\mu} \rho \frac{k^{2}}{\varepsilon}
\end{gathered}
$$

Turbulence is modeled according the RNG $k-\varepsilon$ model:

$$
\begin{aligned}
& \frac{\partial}{\partial t}(\rho k)+\frac{\partial}{\partial x_{i}}\left(\rho k u_{i}\right)=\frac{\partial}{\partial x_{j}}\left[\alpha_{\mathrm{k}} \mu_{e f f} \frac{\partial k}{\partial x_{j}}\right]+G_{k}+G_{b}-\rho \varepsilon-Y_{M}+S_{k} . \\
& \frac{\partial}{\partial t}(\rho \varepsilon)+\frac{\partial}{\partial x_{i}}\left(\rho \varepsilon u_{i}\right)=\frac{\partial}{\partial x_{j}}\left[\alpha_{\varepsilon} \mu_{\mathrm{eff}} \frac{\partial k}{\partial x_{j}}\right]+\frac{\mathrm{C}_{1 \varepsilon} \varepsilon}{k}\left(G_{k}+C_{3 \varepsilon} G_{b}\right)-\frac{\mathrm{C}_{2 \varepsilon} \rho \varepsilon^{2}}{k}-R_{\varepsilon}+S_{\varepsilon} .
\end{aligned}
$$


In these equations $k$ is the turbulence kinetic energy, $\varepsilon$ is the dissipation rate, $G_{k}$ and $G_{b}$ represent the turbulence kinetic energy that originates from velocity gradients and from buoyancy effects respectively, $Y_{M}$ stands for the fluctuating dilation in compressible turbulence. $C_{1 \varepsilon}, C_{2 \varepsilon}$ and $C_{3 \varepsilon}$ are constants. $\alpha_{k}$ and $\alpha_{\varepsilon}$ are the turbulent Prandtl numbers. $S_{k}$ and $S_{\varepsilon}$ are source terms. $R_{\varepsilon}$ is a term deriving from the renormalization group theory that characterizes the RNG model.

These equations are valid only in simulations involving one phase. In order to model multiphase problems numerically, an additional balance equation to consider the presence of two or more phases must be added to the previous equation. By exploiting the VOF model [37], which is based on the definition of the scalar quantity volumetric fraction representing the percentage of one fluid in every cell of the domain, the multiphase problem can be solved. The equation of the volumetric fraction can be expressed as follows:

$$
\frac{\partial \alpha}{\partial t}+\nabla(\alpha \boldsymbol{u})=0
$$

The properties $\Theta$ of the different fluids (such as density and viscosity) are taken to define the properties of an equivalent fluid as follows:

$$
\Theta=\Theta \alpha+\Theta(1-\alpha)
$$

where $\Theta$ represents the generic property of each fluid.

The MULES (Multidimensional Universal Limiter with Explicit Solution) [38] correction can be added in the solver algorithm in order to obtain a more stable and bounded solution of the volumetric fraction field. This is accomplished by adding a dummy velocity field $\left(\boldsymbol{u}_{\boldsymbol{c}}\right)$ in the conservation equation of the volumetric fraction:

$$
\frac{\partial \alpha}{\partial t}+\nabla(\alpha \boldsymbol{u})+\nabla\left(\boldsymbol{u}_{c} \alpha(1-\alpha)\right)=0
$$

An additional source term $\left(S_{g}\right)$ must be added to the equation to account for additional phenomena as cavitation and aeration:

$$
\left.\frac{\partial \alpha}{\partial t}+\nabla(\alpha \boldsymbol{u})+\nabla\left(\boldsymbol{u}_{c} \alpha(1-\alpha)\right)\right)=S_{g}
$$

To calculate the source term, a mathematical model must be introduced. The most used ones for describing cavitation are those by Kunz [39], Merkle [40] and Saurer [41] while aeration can be described using the model by Hirt [42]. In the latter, air is entrained into the water when turbulent energy per unit volume is larger than the stabilizing force of gravity and surface tension per unit volume. The expression for this source term is given as:

$$
S_{g}=C_{a i r} A_{S} \sqrt{2 \frac{P_{t}-P_{d}}{\rho}}
$$

where $\mathrm{S}_{\mathrm{g}}$ is the volume of air per unit of time, $C_{\text {air }}$ is a calibration parameter, $A_{S}$ the free surface area at each cell, and $P_{t}$ (turbulent forces) and $P_{d}$ (stabilizing forces) are given by:

$$
\boldsymbol{P}_{\boldsymbol{t}}=\rho k,
$$




$$
\boldsymbol{P}_{\boldsymbol{d}}=\rho \boldsymbol{g}_{n} L_{T}+\frac{\sigma}{L_{T}}
$$

where $\boldsymbol{g}_{\boldsymbol{n}}$ is the normal component of the gravity, $\boldsymbol{\sigma}$ is the surface tension, and $L_{T}$ is the turbulence characteristic length scale, expressed as:

$$
L_{T}=C_{\mu} \cdot \sqrt{\frac{3}{2}} \frac{k^{\frac{3}{2}}}{\varepsilon},
$$

where $C_{\mu}=0.085$ for the RNG $k-\varepsilon$ turbulence model. An air volume is added to an element of the computational domain when the stabilizing forces $\boldsymbol{P}_{\boldsymbol{d}}$ are exceeded by the perturbing forces $\boldsymbol{P}_{\boldsymbol{t}}$, according to eqn (11). $C_{\text {air }}$ is the coefficient that appears in the equation of the source term that should be calibrated. In this case it is set to 0.5 . Hirt used this value to validate their model. For most of the cases this value has proven to be reasonable. This coefficient indicates the percentage of the free surface area on which air entrapment occurs.

\subsection{Solver settings}

The PIMPLE (merged PISO-SIMPLE) algorithm was used in the simulations. A convergence criterion of 1e-6 was imposed to all field's variables. The Preconditioned Conjugate Gradient (PCG) solver was used for the pressure solver, while the Gauss-Seidel smooth solver was used for the velocity. The Courant number was limited to 0.5 to ensure the stability of the simulations. The first-order implicit Euler scheme was used for time-stepping. A Total Variation Diminishing (TVD) scheme using the vanLeer limiter was adopted for the convection of the volumetric fraction. The convective fluxes in the turbulence equations were discretized using first order schemes.

\section{RESULTS}

Two test cases were considered to study the behaviour of the solver: a vertical plunging jet and a free fall of a droplet. Each of these can be considered as a benchmark for the solver since they can involve some air entrapment at free surface. The introduction of the new solver is expected to improve the physical representation of the real problem due to its capability to consider the air entrainment at free surface.

\subsection{Vertical plunging jet}

In most cases of aeration, air entrapment occurs at a free surface discontinuity where large velocities gradients come across. For this reason, a test case consisting of a jet penetrating into a water pool is considered to test the behaviour of the solver. In Fig. 1 the computational domain is shown. The geometry was taken from Hirt [42] who used it in his validation tests; the problem was modelled as $2 \mathrm{D}$ (a box with dimensions of $0.5 \times 0.5 \mathrm{~m}^{2}$ ) and the domain was meshed with pure quadrilateral elements (about $25 \mathrm{k}$ cells; the geometry was properly partitioned to increase the elements' density in the contact region between the jet and the free surface).

An inlet velocity of $0.4 \mathrm{~m} / \mathrm{s}$ is applied at the jet patch, where turbulent quantities are also imposed, while the pressure must be calculated. Wall functions are applied to the other walls of the domain regarding the turbulent quantities, while the velocity is set to zero.

In Fig. 2 the comparison between the standard multiphase solver of OpenFOAM ${ }^{\circledR}$ and the new one that considers aeration is shown in terms of volumetric fraction field. Two time steps are reported (initial transitory and stabilized filed at regime). 


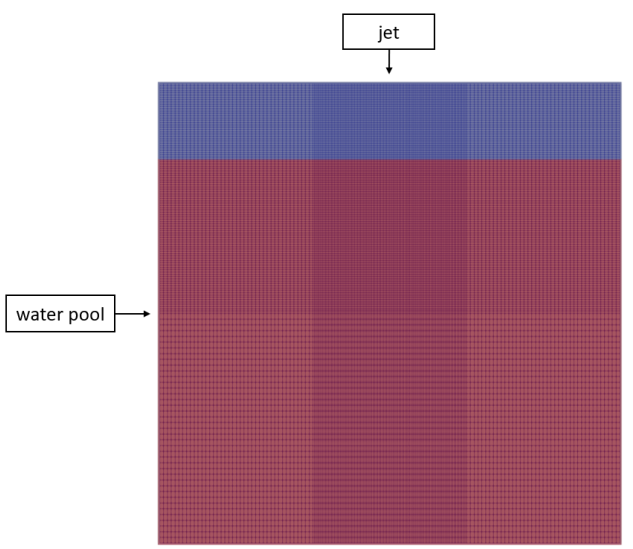

Figure 1: Computational domain. A finer mesh is implemented at the air-water interface.

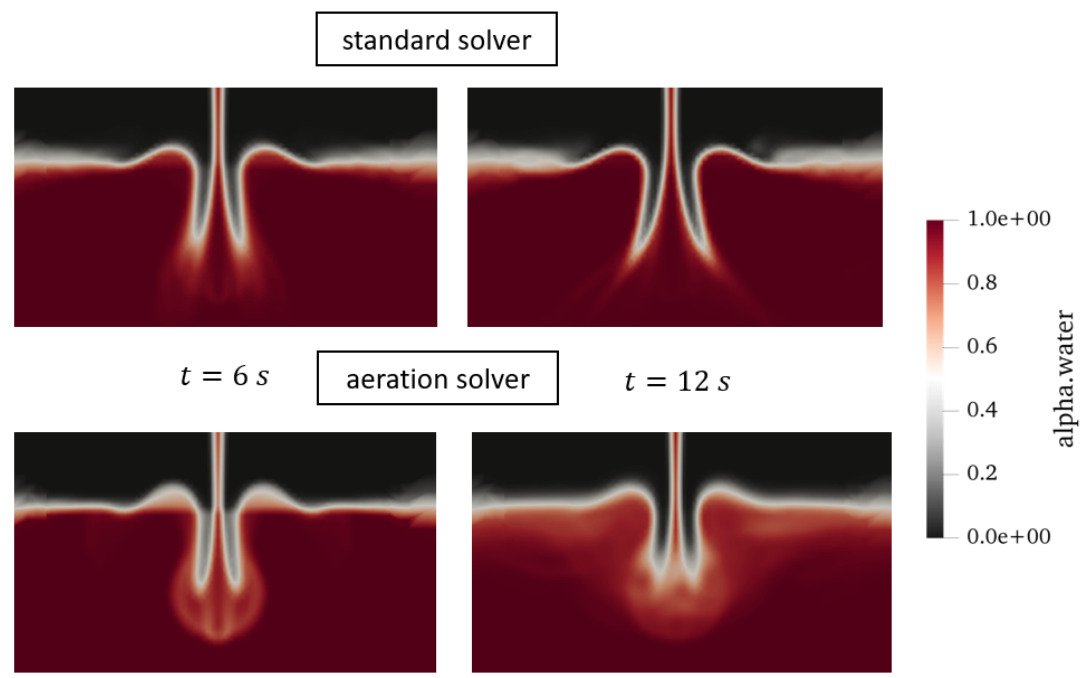

Figure 2: Standard (top row) and aeration (bottom row) volumetric field contour.

It can be observed that the standard multiphase solver promotes a sharp interface between water and air, while the new solver shows a smoother transition at the interface showing that a non-negligible amount of air is entrapped in the water at free surface. This means that, at least qualitatively, the physics of the problem is captured from the solver. Moreover, the contours clearly indicate that, in the case of the standard multiphase solver, the jet substantially enters the steady sump, while the new solver slows down the main flow that tends to diffuse radially due to the presence of foaming effects. Indeed, the foam tends to slow down the water jet penetration forcing it to move laterally.

The effects of the source term at four different time steps are reported in Fig. 3. It can be noticed that it is active on a large region of the free surface, thus indicating that aeration is occurring. 

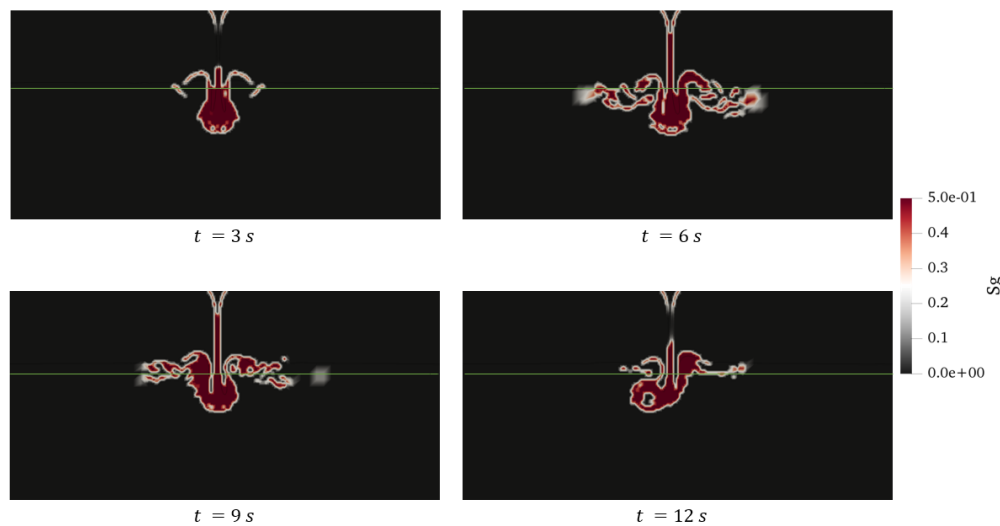

Figure 3: Source term contour at four different time steps.

\subsection{Droplet free fall}

A second test case consisting in a water drop in a pool with the same dimensions of the previous case is considered (Fig. 4).

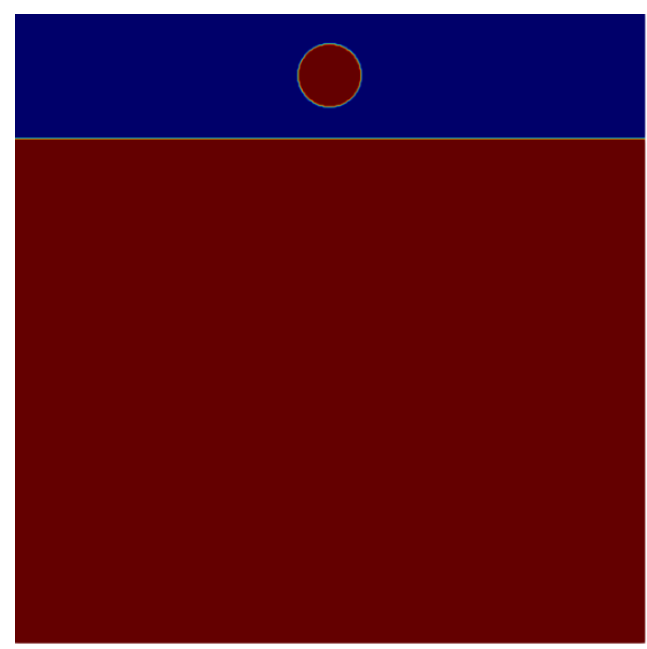

Figure 4: Domain consisting of a water droplet and a water pool.

In this simulation only the gravity is responsible for the droplet movement. In Fig. 5, the volumetric fraction and the effects of the source term are represented.

In this case, aeration seems to be minor with respect to the previous case. In fact, the source term is active on a very small region of the domain and the air trapping is not so evident. This suggests that while in the plunging jet case, the imposition of a flow rate to the fluid forced the aeration phenomenon to take place, in the droplet case the only effect of gravity is not sufficient to cause evident foaming effects at free surface. Therefore, the solver seems to be able to add air entrainment only when it actually occurs $\left(P_{t}>P_{d}\right)$. 


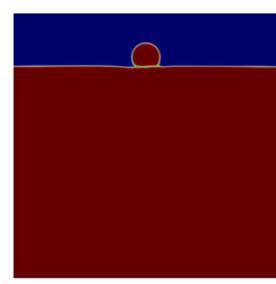

$t=0.02 \mathrm{~s}$
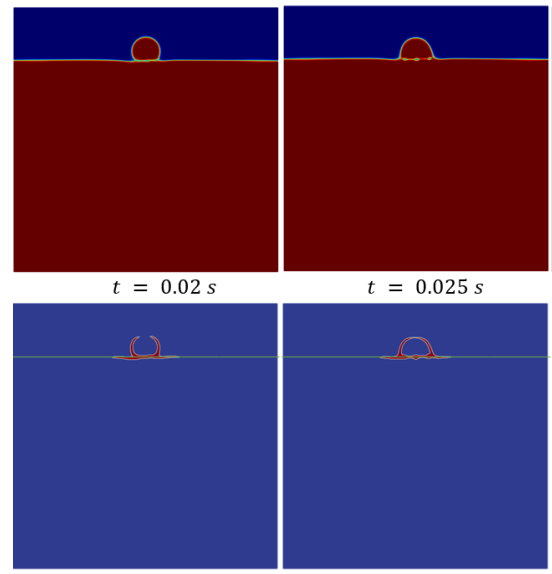

$t=0.025 s$

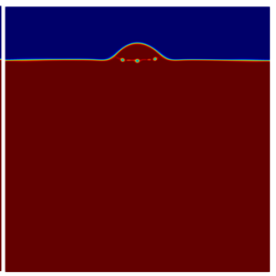

$t=0.03 \mathrm{~s}$
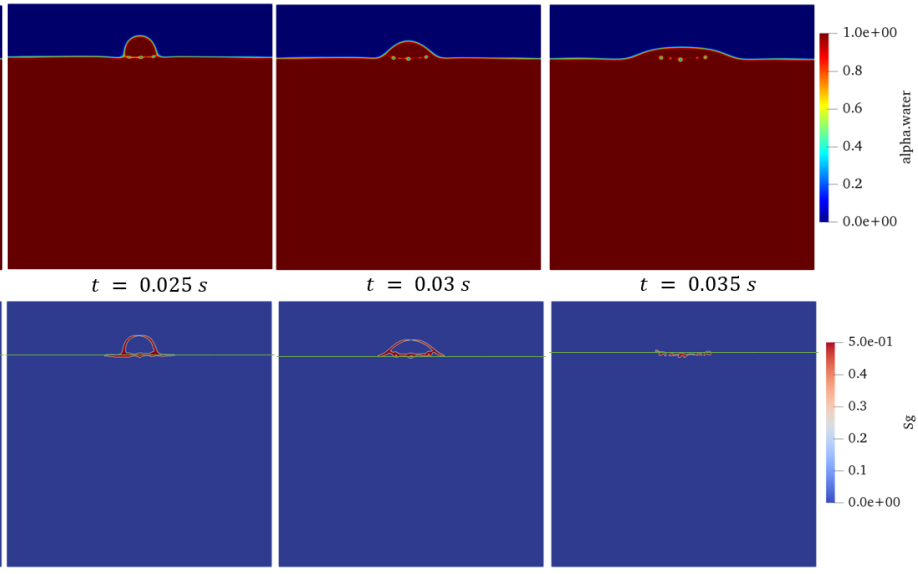

Figure 5: Volumetric fraction and source term contours.

Even if these are relatively simple cases, the solver behaves as expected, at least conceptually, suggesting its being a possible tool for the investigation of the aeration phenomenon. The analysis of the simulations indicates that, at least qualitatively, the solver implemented according to the Hirt model is capable of considering the air entrainment, and, possibly, can be extended to study other hydraulic systems providing at least some design information that would be complex to obtain experimentally.

\section{CONCLUSIONS}

The introduction of CFD has opened new ways in the approach of real engineering problems. Indeed, the benefits of virtual prototypes in terms of cost and time reduction have encouraged designers to exploit simulation tools to support experimental findings. Multiphase problems represent a challenging task in simulations due to the complexity of the required models. In this work, a solver that considers the air entrainment based on the Hirt model is implemented in the open source environment OpenFOAM ${ }^{\circledR}$. The solver was tested on a plunging jet and the results compared with the native multiphase solver of the software used. The results show that, at least qualitatively, the model adds some air at free surface. This solver may be possibly used to analyse operating conditions where the adoption of a standard multiphase solver is not sufficient to capture the generation of aeration. In future, it is planned to apply the solver to different test cases (e.g. to simulate the aeration inside gearboxes).

\section{REFERENCES}

[1] Mastrone, M.N., Hartono, E.A., Chernoray, V. \& Concli, F., Oil distribution and churning losses of gearboxes: Experimental and numerical analysis. Tribology International, 151, p. 106496, 2020.

[2] Burberi, E., Fondelli, T., Andreini, A., Facchini, B. \& Cipolla, L., CFD simulations of a meshing gear pair. Proceedings of the ASME Turbo Expo, vol. 5A-2016, American Society of Mechanical Engineers (ASME), 2016.

[3] Concli, F. \& Gorla, C., CFD simulation of power losses and lubricant flows in gearboxes. American Gear Manufacturers Association Fall Technical Meeting, 2017. 
[4] Concli, F. \& Gorla, C., A CFD analysis of the oil squeezing power losses of a gear pair. International Journal of Computational Methods \& Experimental Measurements, 2, pp. 157-167, 2014.

[5] Concli, F., Maccioni, L. \& Gorla, C., Lubrication of gearboxes: CFD analysis of a cycloidal gear set. WIT Transactions on Engineering Sciences, vol. 123, ed. S. Hernandez, WIT Press: Southampton and Boston, pp. 101-112, 2019.

[6] Concli, F., Schaefer, C.T. \& Bohnert, C., Innovative strategies for bearing lubrication simulations. Preprints, 2019100218, 2019. DOI: 10.20944/preprints201910.0218.v1.

[7] Frosina, E., Senatore, A., Buono, D., Manganelli, M.U. \& Olivetti, M., A tridimensional CFD analysis of the oil pump of an high performance motorbike engine. Energy Procedia, 45, pp. 938-948, 2014.

[8] Ferrari, C. \& Marani, P., Study of air inclusion in lubrication system of CVT gearbox transmission with biphasic CFD simulation. Symposium on Fluid Power and Motion Control, Bath, UK, 2016. DOI: 10.1115/FPMC2016-1767.

[9] Santra, T.S., Raju, K., Deshmukh, R., Gopinathan, N., Paradarami, U. \& Agrawal, A., Prediction of oil flow inside tractor transmission for splash type lubrication. $S A E$ Technical Papers, 2019-01-09, 2019.

[10] Huang, S., Wei, Y., Huo, C. \& Kang, W., Numerical simulation and performance prediction of centrifugal pump's full flow field based on OpenFOAM. Processes, 7(9), 605, 2019.

[11] Concli, F., Low-loss gears precision planetary gearboxes: Reduction of the load dependent power losses and efficiency estimation through a hybrid analyticalnumerical optimization tool. Hochleistungs- und Präzisions-Planetengetriebe: Effizienzschätzung und Reduzierun. Forsch. im Ingenieurwesen/Engineering Res., 81, pp. 395-407, 2017.

[12] Petit, O. \& Nilsson, H., Numerical investigations of unsteady flow in a centrifugal pump with a vaned diffuser. International Journal of Rotating Machinery, 2013, Article ID 961580, 2013. DOI: 10.1155/2013/961580.

[13] Concli, F. \& Gorla, C., Influence of lubricant temperature, lubricant level and rotational speed on the churning power loss in an industrial planetary speed reducer: Computational and experimental study. International Journal of Computational Methods \& Experimental Measurements, 1, pp. 353-366, 2013.

[14] Močilan, M., Husár, Š., Labaj, J. \& Žmindák, M., Non-stationary CFD simulation of a gear pump. Procedia Engineering, 177, pp. 532-539, 2017.

[15] Gao, G., Yin, Z., Jiang, D. \& Zhang, X., Numerical analysis of plain journal bearing under hydrodynamic lubrication by water. Tribololy International, 75, pp. 31-38, 2014.

[16] Sawicki, J. \& Rao, T., Cavitation effects on the stability of a submerged journal bearing. International Journal of Rotating Machinery, 10, pp. 227-232, 2004.

[17] Concli, F., Pressure distribution in small hydrodynamic journal bearings considering cavitation: A numerical approach based on the open- source CFD code OpenFOAM ${ }^{\circledR}$. Lubrication Science, 28, pp. 329-347, 2016.

[18] Concli, F. \& Gorla, C., Numerical modeling of the power losses in geared transmissions: Windage, churning and cavitation simulations with a new integrated approach that drastically reduces the computational effort. Tribololy International, 103, pp. 58-68, 2016.

[19] Riedel, M., Schmidt, M. \& Stücke, P., Numerical investigation of cavitation flow in journal bearing geometry. EPJ Web of Conferences, 45, pp. 1-4, 2013. 
[20] Borges, J.E., Pereira, N.H., Matos, J. \& Frizell, K.H., Performance of a combined three-hole conductivity probe for void fraction and velocity measurement in air-water flows. Experiments in Fluids, 48, pp. 17-31, 2010.

[21] Leandro, J., Bung, D.B. \& Carvalho, R., Measuring void fraction and velocity fields of a stepped spillway for skimming flow using non-intrusive methods. Experiments \& Fluids, 55, pp. 1-17, 2014.

[22] Leprince, G., Changenet, C., Ville, F., Velex, P., Dufau, C. \& Jarnias, F., Influence of aerated lubricants on gear churning losses - An engineering model. Tribology Transactions, 54, pp. 929-938, 2011.

[23] Neurouth, A., Changenet, C., Ville, F., Octrue, M. \& Tinguy, E., Experimental investigations to use splash lubrication for high-speed gears. Journal of Tribology, 139(6), 061104, 2017.

[24] Cerne, G., Peterlin, S. \& Tiselj, I., Coupling of the interface tracking and the two-fluid models for the simulation of incompressible two-phase flow. Journal of Computational Physics, 171, pp. 776-804, 2001.

[25] Drew, D. \& Passman, S., Theory of Multicomponents Fluids, Springer: New York, 1998.

[26] Yan, K. \& Che, D., A coupled model for simulation of the gas-liquid two-phase flow with complex flow patterns. International Journal of Multiphase Flow, 36, pp. 338348, 2010.

[27] Wardle, K.E. \& Weller, H.G., Hybrid multiphase CFD solver for coupled dispersed/segregated flows in liquid-liquid extraction. International Journal of Chemical Engineering, 2013, Article ID 128936, 2013.

[28] Ma, J., Oberai, A., Drew, D., Lahey, R. \& Moraga, F., A quantitative sub-grid air entrainment model for bubbly flows - plunging jets. Computers \& Fluids, 39, pp. 77$86,2010$.

[29] Sene, K.J., Air entrainment by plunging jets. Chemical Engineering Science, 43, pp. 2615-2623, 1988.

[30] Ma, J., Oberai, A., Drew, D., Lahey, R. \& Moraga, F., Modeling air entrainment and transport in a hydraulic jump using two-fluid RANS and DES turbulence models. Heat \& Mass Transfer, 47, pp. 911-919, 2011.

[31] Ma, J., Oberai, A., Drew, D., Lahey, R. \& Moraga, F., A comprehensive sub-grid air entrainment model for RaNS modeling of free-surface bubbly flows. Journal of Computational Multiphase Flows, 3, pp. 41-56, 2011.

[32] Ma, J., Oberai, A., Drew, D., Lahey, R. \& Moraga, F., Two-fluid modeling of bubbly flows around surface ships using a phenomenological subgrid air entrainment model. Computaters \& Fluids, 52, pp. 50-57, 2011.

[33] Bayler, A. \& Emiroglu, M.E., An experimental study of air entrainment and oxygen transfer at a water jet from a nozzle with air holes. Water Environment Research, 76, pp. 231-237, 2014.

[34] Bin, A.K., Gas entrainment by plunging liquid jets. Chemical Engineering Science, 48, pp. 3585-3630, 1993.

[35] Chanson, H., Aoki, S. \& Hoque, A., Physical modelling and similitude of air bubble entrainment at vertical circular plunging jets. Chemical Engineering Science, 59, pp. 747-754, 2004.

[36] Kiger, K.T. \& Duncan, J.H., Air-entrainment mechanisms in plunging jets and breaking waves. Annual Review of Fluid Mechanics, 44, pp. 563-596, 2012.

[37] Hirt, C.W. \& Nichols, B.D., Volume of fluid (VOF) method for the dynamics of free boundaries. Journal of Computational Physics, 39, pp. 201-225, 1981. 
36 Advances in Fluid Dynamics with emphasis on Multiphase and Complex Flow

[38] Rusche, H., Computational Fluid Dynamics of Dispersed Two-Phase Flows at High Phase Fractions, Imperial College of Science, Technology and Medicine: London, 2002.

[39] Kunz, R.F. et al., Preconditioned Navier-Stokes method for two-phase flows with application to cavitation prediction. Computers \& Fluids, 29(8), pp. 849-875, 2000.

[40] Merkle, C.L., Feng, J. \& Buelow, P.E.O., Computational modeling of the dynamics of sheet cavitation. 3rd International Symposium on Cavitation, Grenoble, France, pp. 47-54, 1998.

[41] Saurer, J., Instationären kaviterende Sträömung - Ein neues Modell, basierend auf Front Capturing (VoF) and Blasendynamik, Universität Karlsruhe, 2000.

[42] Hirt, C.W., Modeling Turbulent Entrainment of Air at a Free Surface, Flow Science Report 01-12, Flow Science, Inc., 2003. DOI: 10.1061/40737(2004)187. 